\section{New home for BDNF}

\author{
By Kai-Jye Lou, Staff Writer
}

BDNF's clinical track record has been less than stellar and includes a Phase III failure in amyotrophic lateral sclerosis. A group at the University of California, San Diego attributes past failures to ineffective drug delivery that didn't get enough of the growth factor to the proper locations. The group is trying a gene delivery approach in Alzheimer's disease and has demonstrated BDNF's neuroprotective effects across a panel of rodent and primate models. ${ }^{1}$

By targeting the entorhinal cortex region of the brain, the team showed that brain-derived neurotrophic factor (BDNF) prevented neurodegeneration and decreased cognitive deficits in multiple models of AD.

BDNF has neuroprotective effects in the mammalian brain. The growth factor is produced by neurons in the entorhinal cortex and is then trafficked to the hippocampus-a structure important for learning and memory. ${ }^{2}$

The entorhinal cortex is one of the earliest casualties of $\mathrm{AD}$-neurons in that area degenerate during the initial stages of the disease. ${ }^{3}$ In addition, BDNF levels in the entorhinal and hippocampal regions decline with $\mathrm{AD}$ progression. ${ }^{4}$

With these relationships in mind, Mark Tuszynski and colleagues sought to show that the neuroprotective effects of BDNF could slow the progressive neurodegeneration that occurs in AD. Tuszynski is director of the Center for Neural Repair and a professor of neurosciences at UCSD. He is also a cofounder and director of Ceregene Inc. and a neurologist at the VA San Diego Medical Center in La Jolla.

Ceregene's CERE-110, an adeno-associated viral (AAV) vector encoding nerve growth factor (NGF), is in Phase I testing to treat and prevent $\mathrm{AD}$.

In a paper published in Nature Medicine, a research group led by Tuszynski used lentivirus-mediated gene delivery to increase BDNF in the entorhinal cortex. In six AD models, including mice, rats and monkeys, higher BDNF consistently protected against synapse loss, neuronal death and atrophy. Control groups showed no such benefits.

Increasing $\mathrm{BDNF}$ also improved $\mathrm{AD}$-associated deficits in spatial learning and memory. In the $\beta$-amyloid $(\mathrm{A} \beta)$ rodent $\mathrm{AD}$ models, the improvements in synaptic and behavioral measures occurred independently of effects on $A \beta$ levels.

"The data in the Nature Medicine paper suggest that BDNF could treat the disease through a mechanism directly targeting cell death pathways, independent of $\beta$-amyloid load," said Tuszynski. "BDNF broadly modifies neuronal death mechanisms and improves cellular functional pathways, providing a potential opportunity to synergize with the $\mathrm{AD}$ drugs that are being developed that target amyloid."

\section{Early to the party}

Elliott Mufson, a professor of neurological sciences at Rush University Medical Center, said neurotrophic factor-based therapies like BDNF may be able to affect AD in its earliest stages. "The neurons in the entorhinal cortex are known to undergo degeneration very early in the Alzheimer's disease, and if you can prevent this, you may be able to slow the progression of the disease," he said.

Tuszynski agreed. "The hope is that we can get in early enough to delay disease progression," he said.

William Pardridge had a slightly different view. He thinks BDNF and other neurotrophic factors would be better suited for use later in the disease. "I would imagine a potential two-stage therapy where the first stage involves administering an amyloid disaggregation compound to the brain to remove the plaques," he said. "This can then be followed in a second stage involving the delivery of a neurotrophic factor to the brain to repair the dystrophic neurites," which are the degenerating outgrowths from neurons.

Pardridge is a professor of medicine at the University of California, Los Angeles and CSO of ArmaGen Technologies Inc. The company's AGT-160, an anti- $\beta$-amyloid therapeutic fusion protein, is in preclinical testing for AD. The company also has neurotrophic factor-based therapeutic fusion proteins in preclinical development for stroke and Parkinson's disease (PD).

\section{Special delivery}

Regardless of when BDNF is given in the course of AD, Tuszynski thinks the gene therapy approach will be able to deliver enough of the neurotrophic factor to actually achieve efficacy.

Lack of efficacy has dogged virtually all neurotrophic factors that have entered the clinic. ${ }^{5}$ In 1999, Amgen Inc. discontinued its intraventricular glial cell-derived neurotrophic factor (GDNF) to treat PD. Two years later, the company and partner Regeneron Pharmaceuticals Inc. discontinued a BDNF program in ALS.

"I think delivery in effective amounts has been a major failure in all these trials," said William Mobley, a professor in the Department of Neurology and Neurological Sciences at Stanford University School of Medicine. "We were enamored by all the potent data seen in vitro and in animals." As a result, he said, "the necessary preclinical studies, like the design of a feasible delivery system, just didn't happen."

Tuszynski also said the negative clinical trials of neurotrophic factors "failed to deliver sufficient amounts of these proteins to degenerating neurons." 


\section{TARGETS \& MECHANISMS}

He noted that other researchers have shown that directly infusing a neurotrophic factor-NGF-into the spinal fluid space of the brain of three $\mathrm{AD}$ patients was ineffective and led to widespread dissemination of the protein, which caused pain, nausea and weight loss. ${ }^{6}$

"The real challenge has been getting effective doses of BDNF to the brain and preventing toxicity when growth factors widely spread throughout the brain," Tuszynski told SciBX. "The key is being able to deliver an adequate dose of the factor and restrict the dosing to the degenerating neurons in the disease."

Indeed, last November, Ceregene's CERE-120 failed in a Phase II trial to treat PD. CERE-120 is an AAV type 2 vector encoding the gene for the neurotrophic factor neurturin (NTN).

Despite the setback, Tuszynski thinks gene therapy is still well suited to treat $\mathrm{AD}$ and other neurodegenerative diseases.

In 2005, he and his colleagues reported long-term follow-up data from a Phase I trial showing that ex vivo NGF gene delivery in eight patients with mild $\mathrm{AD}$ had no long-term adverse effects. ${ }^{7}$ Tuszynski said safety and imaging data from that study supported the case for using a gene delivery-based approach for neurotrophic factor-based therapies.

\section{Is BDNF enough?}

Although the work in the Nature Medicine paper suggests therapeutic potential for $\mathrm{AD}$, companies and academics think more validation and proof-of-concept experiments are needed before advancing into the clinic.

"We have not yet seen proof of concept in humans showing that if we provide excess BDNF, we will be able to treat this disease," said Eldad Melamed, chief medical advisor at BrainStorm Cell Therapeutics Inc. and a professor of neurology at Tel Aviv University. "Right now, we still don't know the exact causes of neurodegeneration in diseases like Alzheimer's, Parkinson's and Lou Gehrig's. Once we know what causes the death of these nerve cells, we may be able to design more clever strategies to treat these diseases."

Because many neurodegenerative diseases have unknown causes, BrainStorm is taking a basket approach. The company harvests a patient's bone marrow and isolates the mesenchymal stem cells. Those cells are differentiated in serum-free media into cells that secrete multiple neurotrophic factors.
Stanford's Mobley wanted to see a study demonstrating that AD symptoms can be improved via the BDNF pathway. He also wanted to see additional imaging and animal studies to evaluate whether BDNFbased therapies can increase cortical function.

"Many neurons are involved in $\mathrm{AD}$, and not all are necessarily responsive to BDNF," he said.

Rush Medical's Mufson said he wanted to see better targeting of the BDNF-based therapy to the entorhinal cortex. He also wanted to see $\mathrm{BDNF}$ evaluated in monkeys that overexpress $\mathrm{A} \beta$, as opposed to just rodents.

Tuszynski said his group is evaluating its BDNF gene delivery approach in long-term safety and dosing studies in animal models of AD. He did not give a time frame for starting the Phase I study.

Tuszynski said a patent covering the use of BDNF in AD is pending. The technology is available for licensing from the University of California, San Diego Technology Transfer Office.

Lou, K.-J. SciBX 2(8); doi:10.1038/scibx.2009.301

Published online Feb. 26, 2009

\section{REFERENCES}

1. Nagahara, A.H. et al. Nat. Med.; published online Feb. 8, 2009; doi:10.1038/nm.1912

Contact: Mark H. Tuszynski, University of California, San Diego, La Jolla, Calif.

e-mail: mtuszynski@ucsd.edu

2. Yan, Q. et al. J. Comp. Neurol. 378,135-137 (1997)

3. Price, J.L. et al. Arch. Neurol. 58, 1395-1402 (2001)

4. Hock, C. et al. Arch. Neurol. 57, 846-851 (2000)

5. Dingwell, A. BioCentury 12(31), A10; July 12, 2004

6. Tuszynski, M.H. Lancet Neurol. 1, 51-57 (2002)

7. Tuszynski, M.H. et al. Nat. Med. 11, 551-555 (2005)

COMPANIES AND INSTITUTIONS MENTIONED

Amgen Inc. (NASDAQ:AMGN), Thousand Oaks, Calif. ArmaGen Technologies Inc., Santa Monica, Calif. BrainStorm Cell Therapeutics Inc. (OTCBB:BCLI), New York, N.Y. Ceregene Inc., San Diego, Calif.

Regeneron Pharmaceuticals Inc. (NASDAQ:REGN), Tarrytown, N.Y.

Rush University Medical Center, Chicago, III.

Stanford University School of Medicine, Stanford, Calif.

Tel Aviv University, Tel Aviv, Israel

University of California, Los Angeles, Calif

University of California, San Diego, La Jolla, Calif.

VA San Diego Medical Center, La Jolla, Calif. 